\title{
Discrete Average of Two-Dimensional Shapes ${ }^{\star}$
}

\author{
Isameddine Boukhriss, Serge Miguet, and Laure Tougne \\ Laboratoire LIRIS, Université Lyon 2, \\ 5 avenue Pierre Mendès 69500 , France \\ isameddine.boukhriss@liris.cnrs.fr \\ http://liris.cnrs.fr
}

\begin{abstract}
In this article we present an algorithm for computing discrete average of $\mathrm{n}$ two-dimensional shapes. Our previous work was limited to two shapes, we generalize it to an arbitrary number of objects with consideration of increasing inter-individual variability. The first step of our approach performs a rigid transformation that aligns the shapes as best as possible. The next step consists in searching the progressive metamorphosis of one object toward the other one, that iteratively adds or suppresses pixels. This process is then iterated between the last average shape obtained and the new object from the set according to weighting consideration. It considers the rank in which each shape is added and gives criteria of optimization in variability and global topology preservation. The basic operations are based on geodesic distance transformations and lead to an optimal (linear) algorithm.
\end{abstract}

\section{Introduction}

Electronic devices produce a lot of images in medical, multimedia and physics domains. These images are produced every moment and their interpretation is a very hard and heavy task. It would be of great interest to concentrate all the data in a flexible representative.

Morphing techniques allow the creation of an image starting from an initial image under particular constraints and permit also the generation of a sequence of images starting from given images. This later functionality interests us in this study. An average shape could be among the images in this sequence. In this paper, our goal is to generalize an already developed study of progressive deformation, from one object to another one, to a set of shapes. By considering pair wise shapes, our method is decomposed into two steps: the first one consists in making a rigid registration of the two objects and the second one in computing the deformation. The new obtained average shape is then updated with another shape from the hole set by repeating the same process with proportionality considerations.

\footnotetext{
* This work is supported by the Ragtime project of the Rhone Alpes region and Medigrid project of ACI GRID Program.
} 


\section{State of the Art}

Morphing techniques allow the transformation of a source into a target object. They generate a sequence of images starting from two given images. It is very interesting to investigate this sequence to extract an average shape. Many approaches for morphing are studied.

One of the oldest technique 1 called mesh morphing consists in superimposing a deformable grid on the image source, and to deform this grid so that its intersections indicate particular features of the image. The operation is applied to the image destination, and the transformation of the passage of a grid deformed with the other is calculated by interpolation. Another approach was later introduced [2, based on the mapping of segments, defined in each image by the user. Physical studies gave also another technique based on deformation of a model according to laws of rigidity and elasticity [5]. The best results were observed with point-based morphing. It operates directly on vertices, their interpolation can be done with different methods based on thin plate splines or Gaussian [3] or elastic splines [4]. Our technique consists in adding pixels to one shape and deletion of others from the second with a control technique which makes it possible to generate a sequence of transitions from shapes.

A recent study [6] computes the average shape between two continuous shapes. First, it makes the registration of the two images and it computes the skeleton of the difference between the two shapes. Using an elimination process, it only keeps the points of the skeleton that are equidistant of two borders of two different objects. The method we present in this paper is a generalized discrete version of the previous one. The generalization we propose allows to compute not only a median shape but also the different intermediate shapes.

\section{Preliminaries}

Let us give the formal context of our study and recall some basic notions concerning the inertia moments and neighborhood properties.

\subsection{Neighborhood, Connectivity and Distance}

We consider $2 \mathrm{D}$ shapes in the $Z^{2}$ space. The pixels of the shape have the value 1 and the pixels that belong to the background have the value 0 . The object is considered as 8-connected and background is considered as 4-connected. We work in $3 \times 3$ neighborhood. Let $a$ and $b$ denote two binary shapes, we denote the symmetric difference by $a \Delta b=\{a \cup b\} \backslash\{a \cap b\}$.

In our study, we will use the chamfer distance 3-4 which is a good approximation of the Euclidean distance.

\subsection{Inertia Moments: Eigen Values and Vectors}

In order to make the registration, we will use the moments associated to the shapes. Such descriptors are especially interesting in order to determine the 
position, the orientation and scale of an object. Moreover, one of their main advantages is their small sensitivity to noise. With these moments, we deduce the eigenvectors $V_{1}$ and $V_{2}$ and the associated eigenvalues $\lambda_{1}$ and $\lambda_{2}$ for each shape. Let us suppose $\lambda_{1}>\lambda_{2}$. $V_{1}$ represents the maximal elongation axis of the object. These data will be used to apply the different transformations in the morphing process.

\section{Discrete Average Shape}

\subsection{Previous Work}

The overall system structure of the proposed approach is similar to our previous work [7]. The method is based on aligning rigidly the two considered input shapes and then applying the morphing. The first step is directly deduced from the computation of the inertia moments and consists in a translation and a rotation.

The scaling and re-sampling operations are very important. We have chosen a compromise which aligns the principal vectors on a new system of coordinates and which chooses an intermediate scale between the two shapes (it reduces the biggest and increases the smallest). Lets suppose that our first two shapes are $a$ and $b . \lambda_{a}^{1}$ and $\lambda_{b}^{1}$ are the eigenvalues corresponding to the two maximal elongation of shapes $V_{a}^{1}$ and $V_{b}^{1}$. The factor of scaling of the first shape is:

$$
F_{1}=\sqrt{\left(\lambda_{1}^{a}+\lambda_{1}^{b}\right) / 2 \lambda_{1}^{a}}
$$

The factor of scaling of the second shape is similar and proportional to its maximal elongation:

$$
F_{2}=\sqrt{\left(\lambda_{1}^{a}+\lambda_{1}^{b}\right) / 2 \lambda_{1}^{b}}
$$

Once the two input shapes are superimposed, we deformate one into the other to make morphing. This process is based on two operations: adding and deletion of pixels.

We construct first of all, two kinds of geodesic waves as shown in figure 3 . We denote by $d_{1}$ the 3-4 distance from $a \cap b$ to $a \Delta b$, and $d_{2}$ the 3-4 distance from the complement of $a \cup b$ to $a \Delta b$. Consequently, to each pixel of the difference we associate two distances $d_{1}$ and $d_{2}$. The next step consists in labeling each connected component of $a \Delta b$ in order to permit proportionality considerations between each other.

Let us denote by $\beta$ a parameter, varying between 0 and 1 , that gives the degree of progression of the morphing. To obtain exactly the median shape, the parameter $\beta$ must be equal to $\frac{1}{2}$. Suppose we deform $a$ into $b$.

A pixel of $a \backslash b$ in the $i^{\text {th }}$ connected component of $a \Delta b$ is removed from $a$ if it is labeled with distances $d_{1}$ and $d_{2}$ verifying:

$$
d_{1} \geq d_{1 i}(1-\beta) \text { or } d_{2} \leq d_{2 i} \beta
$$


A pixel of $b \backslash a$ in the $i^{t h}$ connected component of $a \Delta b$ is added to $a$ if it is labeled with distances $d_{1}$ and $d_{2}$ verifying:

$$
d_{1} \leq d_{1 i} \beta \text { and } d_{2} \geq d_{2 i}(1-\beta)
$$

where $d_{j i}$ is the biggest distance value in the $i^{\text {th }}$ connected component according to the $j^{\text {th }}$ propagation. These equations should be modified if we have more than two shapes to average. These considerations will be explained in the following section.

\subsection{N Shapes Generalization}

The important part previously described makes possible the deformation of a shape into another one and to generate their average. This section is about the extension to $\mathrm{n}$ objects. At this level, this extension can be done according at least to two possibilities:

- Dichotomic approach: it consists in subdividing the set of $\mathrm{n}$ objects in $n / 2$ pairs and then applying the same process between each couple. The new $n / 2$ average shapes are then treated to get $n / 4$ other average objects. If the starting whole is odd, we can consider one of the shapes as a first average one.

- Unilateral approach: this approach consists in always keeping the average shape active in all the transformations. That is to say every new object, according to its rank, will directly affect the average form until the last one. We have chosen this approach because it allows to add a posteriori a new shape easily. Suppose we denote $\overline{\mathrm{x}}$ the $(n-1)^{t h}$ average shape created from $(n-1)$ objects, let $x_{n+1}$ be a new form. Suppose we readjusted, scaled and re-sampled the two shapes as best as possible. We suppose that we deform $\overline{\mathrm{x}}$ into $x_{n+1}$. Adding or deletion of pixels will depends on these conditions:

- If we add pixels to $\overline{\mathrm{x}}$ and remove pixels from $x_{n+1}$ :

a pixel of $x_{n+1} \backslash \overline{\mathrm{x}}$ in the $i^{\text {th }}$ connected component of $\overline{\mathrm{x}} \Delta x_{n+1}$ is added to $\overline{\mathrm{x}}$ if it is labeled with distances $d_{1}$ and $d_{2}$ verifying:

$$
d_{1} \leq d_{1 i} \beta \frac{n+1}{n} \text { and } d_{2} \geq d_{2 i}(1-\beta) \frac{n}{n+1}
$$

According to equation 4, we should add more pixels to $\overline{\mathrm{x}}$, so increasing $d_{1 i}$ will be tolerant to accept more pixels. The same reason for decreasing $d_{2 i}$.

- If we add pixels to $x_{n+1}$ and remove pixels from $\overline{\mathrm{x}}$ :

a pixel of $\overline{\mathrm{x}} \backslash x_{n+1}$ in the $i^{\text {th }}$ connected component of $\overline{\mathrm{x}} \Delta x_{n+1}$ is removed from $\overline{\mathrm{x}}$ if it is labeled with distances $d_{1}$ and $d_{2}$ verifying:

$$
d_{1} \geq d_{1 i}(1-\beta) \frac{n}{n+1} \text { or } d_{2} \leq d_{2 i} \beta \frac{n+1}{n}
$$

According to equation 3 , we should remove less pixels from $\overline{\mathrm{x}}$, so decreasing $d_{1 i}$ will be less tolerant to remove pixels. The same reason for increasing $d_{2 i}$. 
One of the important points also, which is in the two approaches, is the order in which the forms are taken. This is closely related to the first phase concerning the scaling. Let us suppose that we have an average form, if the next form which will update it is too small or too large compared to it, we would risk to have a considerable modification on the size of the new average form.

What we have done is according to the maximum elongations of the two forms, we add matter to small shape and remove matter from the biggest. We can also use the same technique based on weighting according to at least two different techniques:

- If there is a sufficient knowledge of the set of forms, we can order them in a growing or decreasing way of variation according to the maximum elongation. This will enable us to have a direction balanced of the growth of our average form each time that a form is selected to update it.

- If the unilateral approach is chosen, which is our case, we use the same factor of weight of the form (its rank in the cycle) which will update the average form like factor loading in the phase of scaling. That is to say, if there is an average form created starting from $n$ forms, the scaling process will be $(n / n+1)$ more attracted towards it than towards the $(n+1)^{t h}$ form. The equations 1 and 2 should be changed. What we did before is equivalent to compute an average between $\lambda_{1}^{a}$ and $\lambda_{1}^{b}$, if we suppose that $\lambda_{1}^{a}$ is now the big eigenvalue of our recent average shape, we should give more weight to its value. So instead of having $\frac{\lambda_{1}^{a}+\lambda_{1}^{b}}{2}$, we will get:

$$
\operatorname{coe} f=\lambda_{1}^{a}+\left|\frac{\lambda_{1}^{a}-\lambda_{1}^{b}}{n+1}\right|
$$

Thus, the factors of scaling will attract the two shapes, according to maximal elongation, to a shape closer to the recent average one.

$$
F_{1}=\sqrt{\operatorname{coef}} / \sqrt{\lambda_{1}^{a}} \quad F_{2}=\sqrt{\operatorname{coef}} / \sqrt{\lambda_{1}^{b}}
$$

Figure 1 gives the result of each average shape obtained during the whole process. It indicates that we can have a considerable size modification if we have important inter-individuality. This not the case in figure 2 where we apply our

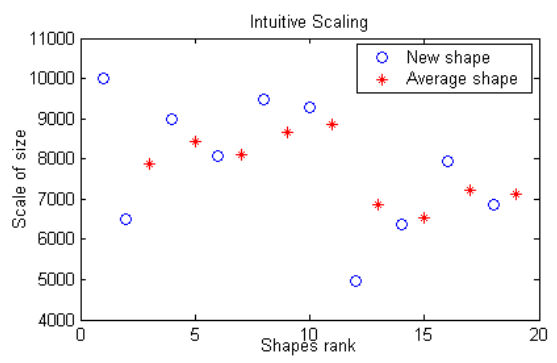

Fig. 1. Scaling with intuitive coefficients(1/2) 
modified coefficients $F_{1}$ and $F_{2}$. Each average shape is more attracted by the previous one even if we introduce a shape with important variation of size.

In order to preserve topology, we adopted a technique which consists in adding only pixels from the difference of the shapes to their intersection. By considering each layer of propagation as a source, we compute the next layer which is connected to the previous one.

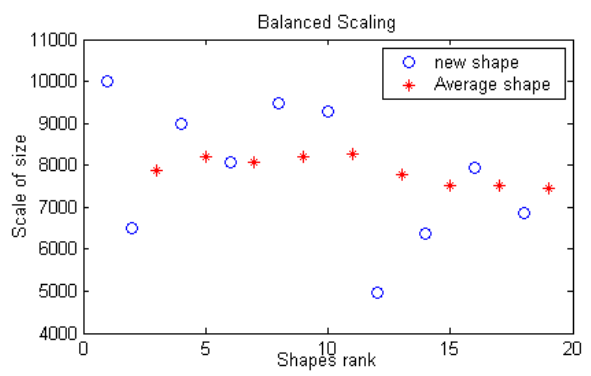

Fig. 2. Scaling with weighting considerations

\section{Results}

In order to validate our approach, we made tests on a set of fishes. They have an important inter-individual variability concerning scale and shape. We begin with two arbitrary shapes that we registrate and scale. We propagate then the two geodesic waves. By fixing the parameter $\beta=1 / 2$ we generate our first average fish. This process was then iterated by considering:

- The order in which each shape is added to update the recent average one.

- The proportionality of adding and deletion of pixels depend on the rank of the new added shape.

We made tests on ten shapes as shown in figure 3. The order in which they were treated was arbitrary chosen. For best check of the whole process, we extracted the two last average shapes obtained as shown in figure 5. We remark, according to the order in which the shapes are treated, that results seem to be satisfying. The variations are small due to the fact that starting from a certain rank, average shape is less influenced by new shapes. It should be noted that an anti-aliasing filter could be applied to improve the results. Figure 4 gives the distance propagation results between the $8^{\text {th }}$ average shape and the last shape to get the $9^{\text {th }}$ one. These results are used to decide about adding or deletion of pixels. Shaded zones of the difference of the two shapes in the figure indicate, according to the final result, that we have small number of added/deleted pixels in connected components where the maximum values due to the propagations are important. However, this number is almost null in the other connected components. It is obvious that the result should be $\frac{1}{10} * \beta$ proportional to the maximum values. The final result is so more attracted to the $8^{\text {th }}$ average shape. 


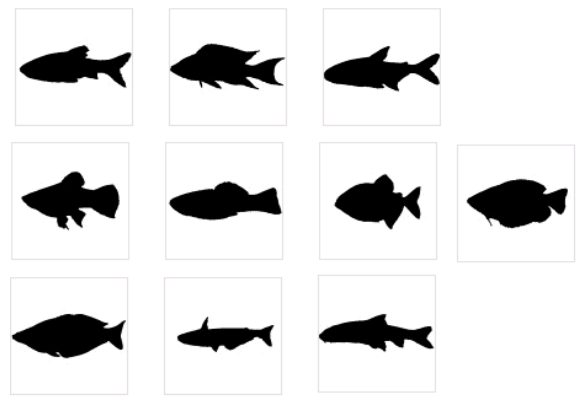

Fig. 3. Sample of our fish data base
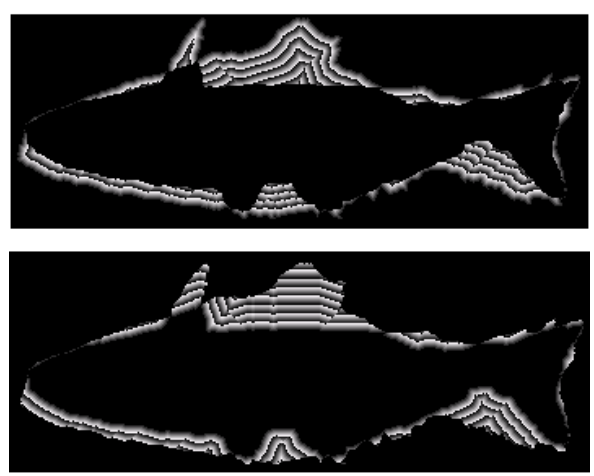

Fig. 4. Distance propagation in the $9^{\text {th }}$ average shape generation process

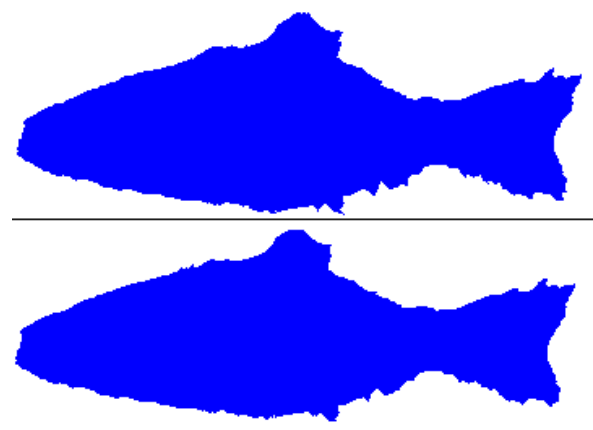

Fig. 5. The $8^{\text {th }}$ and $9^{\text {th }}$ average shape 


\section{Conclusion}

The proposed approach gives a linear solution for computing an intermediate shape between arbitrary input set of binary objects. This average shape could be updated easily. We give also a solution to inter-individual variability by balancing the scaling process in the first step of registration between shapes. This permits less influence of size difference which can affect clearly the average shape even between two iterations. A progression parameter $\beta$ ranging from 0 to 1 allows to control the influence of each input shape.

In this paper we have used the chamfer distance as a good integer approximation of Euclidian distance. An improvement in precision could be the use of the Euclidian distance itself which can also be computed in linear time as shown in [8] at least for 2D domains. With our approach, the topology of intermediate shapes is "globally" preserved, due to the continuous propagation from the intersection of shapes to their difference. An heuristic for reaching better results could be to use thinning or thickening operators, allowing to ensure that the removal or adding of a point does not change the topology of intermediate. These topics and other problems related to noise and segmentation errors should be the subject of further studies.

\section{References}

1. Smythe DB, A two-pass mesh warping algorithm for object transformation and image interpolation, Technical Report 1030, Calif, 1990.

2. T. Beier and S. Neely, Feature-based image metamorphosis, In: Computer Graphics and Interactive Techniques, 1992, pp. 35-42.

3. N. Arad, N. Dyn, D. Reisfeld, and Y. Yeshurin, Image warping by radial basis functions: application to facial expressions. In: CVGIP 56(2), 1994, pp. 161-172.

4. A. Hassanien and M. Nakajima. Image morphing of facial images transformation based on navier elastic body splines. In Computer Animation, volume 7, pages 3-23, 1998.

5. T. Sederberg and E. Greenwood. A physically-based approach to 2-D shape blending. Computer Graphics, vol 26, July 1992,35-42.

6. R. Blanding, G. Turkiyyah, D. Storti, and M. Ganter, Skeleton-based Three Dimensional Geometric Morphing. Computational Geometry, 15, 2000, pp. 129-148.

7. I. Boukhriss, S. Miguet and L. Tougne, Two Dimensional discrete morphing, In: Combinatorial Image Analysis: 10th International Workshop, december 2004, pp. 409-420.

8. D. Coeurjolly, S. Miguet and L. Tougne, 2D and 3D Visibility in Discrete Geometry: An Application to Discrete Geodesic Paths, Pattern Recognition Letters, 2004, vol 25 , pp. $561-570$. 